\title{
Separate roles for executive and phonological components of working memory in mental arithmetic
}

\author{
ANSGAR J. FÜRST and GRAHAM J. HITCH \\ Lancaster University, Lancaster, England
}

\begin{abstract}
A dual-task methodology was used to investigate the roles played by executive and phonological aspects of working memory in mental arithmetic. Experiment 1 showed that suppression of articulation impaired the ability to add a pair of briefly presented three-digit numbers. Suppression had no effect when the need to store temporarily was minimized by making the numbers visible throughout calculation. Experiment 2 showed that disrupting executive processes by requiring concurrent performance of a Trails task impaired the ability to add numbers that remained permanently visible. Performance on the Trails task deteriorated as the number of carry operations in the addition increased. Experiment 3 showed that this decline in Trails performance was not simply due to the extra time taken by carrying. These and other features of the results suggest that the carrying component of mental arithmetic places substantial demands on executive processes, whereas the need to retain problem information is met by the phonological loop. The results are consistent with an interpretation of executive processes according to which there is a limit on the capacity to inhibit strongly primed routine operations.
\end{abstract}

In a recent review, Ashcraft (1995) emphasized the dependence of mental calculation on working memory, the limited-capacity system for keeping track of temporary information during ongoing processing (see, e.g., Baddeley \& Hitch, 1974; Daneman \& Carpenter, 1980). Empirical studies tend to support this view (Ashcraft, Donley, Halas, \& Vakali, 1992; Hitch, 1978; Lemaire, Abdi, \& Fayol, 1996; Logie, Gilhooly, \& Wynn, 1994; but see Butterworth, Cipolotti, \& Warrington, 1996, for an exception). Nevertheless, several models of arithmetic do not mention working memory (see, e.g., Ashcraft, 1982; Campbell, 1995; Dehaene \& Cohen, 1995; Deloche \& Seron, 1987; McCloskey, 1992; Widaman, Geary, Cormier, \& Little, 1989), and relatively little is known about how working memory supports calculation (Logie et al., 1994). The present study took an analytic approach and attempted to identify the role of different components of working memory in major features of mental addition.

The investigation was based on a model of working memory as comprising a central executive and two subsidiary stores (Baddeley \& Hitch, 1974; see also Baddeley, 1986, 1992). Briefly, the central executive is responsible for control processes, including use of the subsidiary stores. Baddeley (1986) equated the executive with the su-

A.J.F. was supported by an ESRC research studentship and is grateful to Christian Marendaz of the LPE, Université Pierre Mendès France (Grenoble), for support during preparation of the manuscript. We thank John Adams and Janet McLean for many helpful discussions and Steve Dewhurst for commenting on the manuscript. Correspondence concerning this article should be addressed to G. J. Hitch, Department of Psychology, Lancaster University, Lancaster LAl 4YF, England (e-mail: g.hitch@lancaster.ac.uk). pervisory attentional system of Norman and Shallice (1980; see also Shallice, 1982), in which intentional control requires the inhibition of competing actions. However, in a recent development, Baddeley (1996) proposed a fractionation of the executive into a number of functions, which included interacting with long-term memory. The subsidiary stores in working memory are specialized for holding and manipulating different types of temporary information. The phonological loop holds rapidly decaying verbal information, which can be refreshed by subvocal rehearsal, whereas the visuospatial sketchpad holds visuospatial information and supports visual imagery.

The Baddeley and Hitch (1974) model allows a consideration of whether different subsystems of working memory are responsible for different aspects of mental arithmetic. For this purpose, it is assumed that calculations involving multidigit numbers typically require a series of steps, each involving the retrieval of an arithmetical fact from long-term memory (Dansereau \& Gregg, 1966; Hitch, 1978; McCloskey, 1992). Evidence from verbal tasks suggests that retrieval from long-term memory does not place heavy demands on working memory (see, e.g., Baddeley, Lewis, Eldridge, \& Thomson, 1984; Conway \& Engle, 1994). However, another feature of stepby-step calculation strategies is that they involve storing interim results and other temporary information. Storing such information would be expected to be a function of either the phonological loop or the visuospatial sketchpad. A third feature of calculation strategies is that they sometimes involve extra operations, such as carrying and borrowing. These operations are of special interest, since they can be regarded as subroutines that require interrupting the normal sequence of operations. Accordingly, 
carrying and borrowing involve inhibiting the tendency to continue the sequence and hence should require supervisory attentional control. The possibility that carrying has special status is suggested by evidence that extended practice in addition reduces the cost of carrying but has little effect on retrieval of number facts from long-term memory (Frensch \& Geary, 1993). Thus, in summary, there are grounds for supposing that different aspects of the calculation process put different demands on the central executive and buffer stores in working memory.

Although sparse, previous work bears on the above conceptual analysis. For example, providing an external record of an arithmetic problem improves performance by reducing the load on temporary storage (Hitch, 1978; see also Adams \& Hitch, 1997). However, it is not clear which component of working memory provides this storage function. Retrieving arithmetical knowledge from longterm memory appears to involve obligatory activation of number facts (LeFevre, Bisanz, \& Mrkonjic, 1988), consistent with a lack of executive involvement. However, other evidence suggests that fact retrieval processes may not be entirely automatic (Zbrodoff \& Logan, 1986; see also Ashcraft et al., 1992) and that processes other than direct retrieval are sometimes used (LeFevre, Sadesky, \& Bisanz, 1996).

A substantial investigation of working memory and mental arithmetic was reported by Logie et al. (1994). A cumulative addition task was used, in which participants were given a series of auditorily or visually presented twodigit numbers and were required to state the final total. Calculations involved either a single carry operation or multiple carries. A dual-task methodology was used, in which addition was combined with various secondary tasks. These included articulatory suppression to disrupt the phonological loop (Baddeley, Thomson, \& Buchanan, 1975), a spatial tapping task to interfere with the visuospatial sketchpad (Baddeley \& Lieberman, 1980), and a random generation task to disrupt the central executive (Baddeley, 1986). Random generation interfered with calculation most, articulatory suppression had a smaller effect, and the spatial task only caused a small impairment when additions were presented visually. Logie et al. inferred major roles for the central executive and the phonological loop in maintaining accuracy in calculation, and a more restricted role for the visuospatial sketchpad. However, their investigation did not satisfactorily resolve whether different subsystems are involved in different aspects of calculation. For example, problems with more carries did not show greater disruption in any of the dual-task conditions. Logie et al. suggested that keeping track of carries does not place much load on working memory. However, their experiment may not have been very sensitive on this point. For example, the manipulation of carrying was not fine-grained.

The present investigation began as a follow-up of Logie et al. (1994), in an attempt to differentiate the roles of executive and phonological processes in arithmetic. There were two principal modifications. First, the manipulation of carrying included problems with different numbers of carry operations, including no carrying at all. It was reasoned that these conditions would give more precise information about the demands placed by carrying. Second, a relatively novel secondary task was used to disrupt the central executive. Logie et al. were unable to analyze performance on their random generation task fully because they did not have long enough runs of responses to assess randomness. Recently, Baddeley (1996) reported using a task based on the Trails test to disrupt executive processes. The Trails test forms part of the HalsteadReitan neuropsychological assessment battery and is an indicator for frontal lobe damage (Lezak, 1983). In one form, numbered and lettered circles have to be joined by alternating between the alphabet and the counting sequence, as in A-1-B-2-C-3- and so forth. At a theoretical level, switching between familiar streams should involve the executive function of inhibiting prepotent responses. Consistent with such an analysis, Baddeley (1996) reported that nonverbal random generation was disrupted by performing an oral Trails task at the same time. The Trails task was adopted in the present study because it is tightly constrained and because assessing performance with it is relatively straightforward. It is also easier to explain to participants than random generation.

Pilot studies suggested that it was too difficult for people to alternate between two familiar verbal sequences at the same time that they were performing complex mental arithmetic. Therefore, in the first experiment we used a simplified Trails-type task. To anticipate, useful information was obtained, but the simplified Trails task proved too easy. Accordingly, further piloting established conditions under which participants were able to do the full Trails task at the same time as arithmetic, and this procedure was adopted in Experiments 2 and 3.

\section{EXPERIMENT 1}

This experiment tested two hypotheses. The first was that the phonological loop is used to store temporary information during arithmetic. To investigate this, participants were required to suppress articulation while doing multidigit additions. Problems either were presented briefly, and therefore had to be remembered in order to complete the answer, or remained visible. It was predicted that occupying the loop would be especially disruptive with brief presentation where there was a higher storage load.

The second hypothesis was that the central executive is responsible for carrying. In the simplified Trails task, participants were given a random letter and recited the alphabet from that point. It was assumed that, despite the absence of a switching component, executive processes would be involved in mentally scanning the alphabet to find the starting point and keeping track (Hamilton \& Sanford, 1978). Accordingly, it was predicted that this recitation task would disrupt calculation more than would 
articulatory suppression and would do so even more when carrying was required.

\section{Method}

\section{Participants}

Thirty students at Lancaster University were paid for their participation. All were native English speakers. Eighteen were tested with a brief presentation of problems, and 12 with a continuous presentation. (Note that the unequal group sizes were an unintended consequence of the way the experiment was run.)

\section{Design and Stimuli}

A factorial design was used, with presentation condition (continuous or brief) as a between-subjects factor and interference (mental arithmetic alone, with suppression, or with recitation) and number of carries (zero, one, or two) as within-subjects factors. Dependent variables were the accuracy and latency of solutions to the problems and performance on the concurrent tasks.

There were 36 addition problems, each consisting of two threedigit numbers that summed to a three-digit answer. These were divided into equal thirds, with zero, one, or two carries. The digit 9 was excluded in order to avoid one type of ambiguous error (e.g., $362+$ $197=569$ could reflect an inappropriate carry or substitution of a digit from the first number into the answer). Problems were arranged in three blocks comprising three practice trials (one of each carry type) and nine experimental trials. Magnitudes of answers were roughly equated between blocks, and the order of experimental problems was randomized within blocks. The participants were randomly assigned to one of the six orders of administering the experimental conditions. Assignment of blocks to conditions was counterbalanced.

\section{Apparatus and Procedure}

Each problem was shown at the center of a computer screen in columnwise Arabic notation in an invisible rectangle $6.5 \mathrm{~cm}$ high and $9.5 \mathrm{~cm}$ wide. In the brief presentation condition, the problem was shown for $4,000 \mathrm{msec}$, followed by a blank screen. (This duration was the time it took to read problems aloud in pilot work.) In the continuous presentation condition, the display was switched to the blank screen after the participant finished responding.

Each trial began with a "Ready" signal. In the recitation condition, the experimenter announced a randomly chosen starting letter. The participant then began reciting the alphabet from that letter at a rate of $1 / \mathrm{sec}$, paced via an auditory metronome. In the articulatory suppression condition, the participant started repeating the word "the" at the same paced rate. In both dual-task conditions, the addition problem was displayed after five spoken responses. In the control condition, the problem was presented when the participant was ready. A timer controlled by PsychLab software was triggered by the onset of the problem and was stopped by a keypress from the experimenter when the participant finished - riting the answer. This method of timing was regarded as sufficiently accurate, given that latencies were of the order of several seconds. The signal for the next trial appeared 1,000 msec later. A separate response sheet was used for each trial.

The participants were tested individually and sat approximately $30 \mathrm{~cm}$ in front of the display. They were told that on each trial, they would see two three-digit numbers that would sum to another threedigit number, and they were asked to write down the total in the order from units to tens to hundreds. They were asked not to make additional notes or to use their fingers. The instructions stressed the importance of accuracy but encouraged participants to guess or pass when they were unsure. There was no time limit on answering and no feedback on accuracy. In dual-task conditions, the participants were required to continue performing the concurrent task until after they had finished the calculation. The participants were reminded whenever necessary of the requirement for maintaining both speed and accuracy on the concurrent task.

\section{Results}

On each trial, concurrent task performance was considered inaccurate if it contained at least one incorrect response or omission (i.e., a response delayed more than one metronome beat). For brief presentation, the proportions of inaccurate trials in the suppression and recitation conditions were $1.9 \%$ and $0.6 \%$, respectively. These trials were excluded from the analysis. With continuous presentation, there were no errors on the concurrent tasks.

Table 1 shows calculation errors scored according to whether or not the entire answer was correct. As was expected, brief presentation was associated with more errors and a bigger effect of articulatory suppression. However, the effect of the recitation task was indistinguishable from that of suppression. A two-way analysis of variance (ANOVA) revealed a significant main effect of presentation condition $[F(1,28)=14.40, p<.001]$, but the main effect of concurrent task and the interaction fell short of significance $[F(2,56)=2.27$ and $F(2,56)=2.56, p<$ .10 , respectively]. Further analyses confirmed that performances in the two dual-task conditions were equivalent with either brief or continuous presentation $\left(F_{\mathrm{S}}<1\right)$. Data from the two dual-task conditions were, therefore, pooled in subsequent comparisons, which showed that there was significant dual-task interference when presentation was brief $[F(1,28)=17.66, p<.001]$ and no interference when presentation was continuous $(F<1)$.

Mean latencies for correct responses in the arithmetic task ranged between 5 and $7 \mathrm{sec}$ and showed no obvious variation across conditions (see Table 1). A two-way ANOVA confirmed that there were no reliable effects of presentation duration $[F(1,28)=2.48]$ or concurrent task $[F(2,56)=1.20]$ and no interaction $[F(2,56)=1.91]$.

Table 2 shows calculation errors for the brief presentation condition broken down by number of carries. Errors increased with the number of carries but were at or near floor for no-carry problems. Accordingly, data were analyzed using a $2 \times 3$ ANOVA, with number of carries

Table 1

Mental Arithmetic Performance (With Standard Deviations) in Experiment 1

\begin{tabular}{|c|c|c|c|c|}
\hline \multirow[b]{2}{*}{ Condition } & \multicolumn{2}{|c|}{ Errors $(\%)$} & \multicolumn{2}{|c|}{ RT ( $\mathrm{sec})$} \\
\hline & $M$ & $S D$ & $M$ & $\overline{S D}$ \\
\hline \multicolumn{5}{|l|}{ Brief presentation } \\
\hline Control & 16.0 & 13.9 & 5.5 & 1.7 \\
\hline $\begin{array}{l}\text { Arithmetic with } \\
\text { suppression }\end{array}$ & 32.4 & 25.4 & 5.4 & 1.5 \\
\hline $\begin{array}{l}\text { Arithmetic with } \\
\text { recitation }\end{array}$ & 31.7 & 20.7 & 5.4 & 1.1 \\
\hline $\begin{array}{l}\text { Continuous presen } \\
\text { Control } \\
\text { Arithmetic with }\end{array}$ & 10.2 & 12.9 & 5.8 & 2.3 \\
\hline $\begin{array}{l}\text { suppression } \\
\text { Arithmetic with }\end{array}$ & 9.3 & 11.4 & 6.6 & 2.8 \\
\hline recitation & 10.2 & 12.0 & 7,0 & 3.3 \\
\hline
\end{tabular}


Table 2

Errors in the Brief Presentation Condition as a Function of Number of Carries (With Standard Deviations)

\begin{tabular}{|c|c|c|c|c|c|c|}
\hline \multirow[b]{3}{*}{ Condition } & \multicolumn{6}{|c|}{ Errors $(\%)$} \\
\hline & \multicolumn{2}{|c|}{ No Carry } & \multicolumn{2}{|c|}{ One Carry } & \multicolumn{2}{|c|}{ Two Carries } \\
\hline & $M$ & $\overline{S D}$ & $M$ & $\overline{S D}$ & $M$ & $S D$ \\
\hline Control & 0 & & 22.2 & 28.0 & 25.9 & 29.3 \\
\hline $\begin{array}{l}\text { Arithmetic with } \\
\text { suppression }\end{array}$ & 7.4 & 24.4 & 35.2 & 26.7 & 53.7 & 41.4 \\
\hline $\begin{array}{l}\text { Arithmetic with } \\
\text { recitation }\end{array}$ & 1.9 & 7.9 & 37.0 & 37.7 & 55.6 & 34.3 \\
\hline
\end{tabular}

(one vs. two) and interference condition (control, suppression, or recitation) as factors. This revealed main effects of number of carries $[F(1,17)=5.93, p<.05]$ and concurrent task $[F(2,34)=4.39, p<.05]$ but no interaction $[F(2,34)=1.15]$.

Errors on individual digits were classified as forgotten carries (e.g., $245+162=307$ ), inappropriate carries (e.g., $356+213=579$ ), substitutions of a problem digit into the answer (e.g., $123+561=681$ ), or other errors. Proportions of these categories were computed separately for each syntactical position (hundreds, tens, and units) and then averaged. In the brief presentation condition, the first three categories together accounted for $40.7 \%$ of all errors. Forgotten carries were most frequent (23.2\%), followed by inappropriate carries $(10.6 \%)$ and problem/ answer substitutions $(6.9 \%)$.

\section{Discussion}

The simplified Trails task turned out to be much less demanding than had been anticipated and did not have a different effect from articulatory suppression. The experiment was therefore uninformative about the involvement of executive processes in carrying.

The aim of investigating whether the phonological loop is used to store problem information was achieved by comparing the effects of concurrent articulation on solving brief and continuously presented problems. Comparisons based on data pooled over the two interference conditions indicated that concurrent articulation resulted in increased errors of calculation when problems were presented briefly, but not when they were continuously visible. This is consistent with the hypothesis that the phonological loop is recruited when problem information has to be stored. Note that the present data underestimate the importance of the phonological loop, because faster participants would be able to complete some of the calculation while a briefly presented problem was visible, thereby reducing the load on temporary information storage.

The results also have implications for the role of working memory in retrieving factual arithmetical knowledge from long-term memory. Thus, finding that irrelevant articulation did not disrupt calculation when problems were continuously visible indicates that the phonological loop is not involved in these processes.
In summary, the phonological loop appears to be involved in maintaining problem information in mental arithmetic, but not in retrieving factual knowledge. However, the results were uninformative about the role of executive processes, most likely because the modified Trails task was insufficiently demanding. Accordingly, the next experiments reverted to the initial plan of using the full Trails task of alternating between two familiar sequences to disrupt executive processes.

\section{EXPERIMENT 2}

Further pilot work indicated that participants could combine the full Trails task with arithmetic, provided that Trails responses were not paced too rapidly and problems were visible throughout the calculation. Experiment 2 therefore involved two dual-task conditions in which participants solved continuously presented arithmetic problems while either performing the full Trails task or suppressing articulation. There was also a control condition, in which there was no dual task. As before, the suppression task provided a control for the articulatory component of the Trails task and was paced at the same rate so as to equate tasks for the total amount of articulation.

\section{Method}

\section{Participants}

Twenty-four students at Lancaster University volunteered. All were native English speakers and were paid for their participation.

\section{Design}

A two-way within-subjects factorial design was used. Factors were experimental condition (arithmetic alone, arithmetic with suppression, or arithmetic with Trails) and number of carries (zero, one, or two). The dependent variables were the accuracy and latency of arithmetic solutions and a measure of concurrent task performance.

\section{Stimuli}

Mental arithmetic. The stimuli were a set of 45 additions conforming to the same constraints as in Experiment 1 . In one-carry problems, the carry was equally often in the tens or hundreds. Each experimental condition consisted of three practice problems (one of each carry type), followed by a block of 12 experimental problems presented in a random order. Details of counterbalancing were as before.

Trails task. On each trial, the participants heard one of the first seven letters in the alphabet and a day of the week (e.g., C-Thursday). These starting points were independently randomized.

\section{Apparatus and Procedure}

These were similar to those in the first study. In all the conditions, addition problems remained visible until participants had completed their written answers. On each trial, the starting items for the Trails task were read out by the experimenter. The participant repeated them and began reciting the two sequences in alternation from that point (i.e., D-Friday, E-Saturday, etc.). The participants were told that if they lost their place, they were to restart with an arbitrary pair (while avoiding repetitions of the same pair). They were also told that if they came to the end of a sequence, they should return to the beginning (i.e., from $\mathrm{Z}$ to $\mathrm{A}$ and from Sunday to Monday). In both the Trails and the suppression conditions, articulation 
was paced by an auditory metronome set at a period of $1.2 \mathrm{sec}$, and the addition problem was presented after four responses. All other details were the same as those in Experiment 1.

\section{Results}

\section{Mental Addition}

As before, calculation answers were scored according to whether they were correct or incorrect. Mean error percentages show that the Trails task caused substantial interference (see Table 3 ). An ANOVA revealed a highly significant effect of conditions $[F(2,46)=45.44, p<.001]$, with significantly more errors in the Trails condition than in either the control or the suppression condition $[F(1,23)=61.85, p<.001$, and $F(1,23)=46.89, p<.001$, respectively]. There was a small increase in errors in the suppression condition, relative to the control condition, that just failed to reach significance $[F(1,23)=3.93, p<.06]$.

Mean latencies for correct solutions followed a similar pattern (Table 3 ). The effect of interference condition was highly significant $[F(2,46)=42.71, p<.001]$. Pairwise comparisons showed that significantly more time was required to complete answers in the Trails condition than in either the control or the suppression condition $[F(1,23)=$ $40.85, p<.001$, and $F(1,23)=46.41, p<.001$, respectively]. However, there was no significant difference between the control and the suppression conditions.

A breakdown of errors according to the amount of carrying showed that most of the interfering effect of Trails was associated with one- and two-carry problems (see Table 4). Because distributions were markedly nonnormal, nonparametric statistics were used to assess interference effects. The increase in errors with number of carries in the Trails condition was highly significant $(p<.001$, Friedman test), but trends in the other conditions were nonsignificant (suppression, $p=.08$; control, n.s.).

The percentages of different types of errors in answers were calculated as in Experiment 1. Taken together, the three defined categories accounted for $67.3 \%$ of all errors. Errors of forgetting to carry were the most frequent $(50.7 \%)$, followed by errors of carrying inappropriately $(10.0 \%)$ and problem/answer substitutions $(6.6 \%)$.

\section{Concurrent Task Performance}

Tape recordings were used to score concurrent task performance. Performance in the interval between successive metronome beats was counted as accurate as long as an appropriate response was made. By this criterion, the suppression task was performed with $100 \%$ accuracy on

Table 3

Mental Arithmetic Performance (With Standard Deviations) in Experiment 2

\begin{tabular}{cccccc}
\hline & \multicolumn{2}{c}{ Errors (\%) } & & \multicolumn{2}{c}{ RT (sec) } \\
\cline { 2 - 3 } \cline { 5 - 6 } Condition & $M$ & $S D$ & & $M$ & $S D$ \\
\hline $\begin{array}{c}\text { Control } \\
\text { Arithmetic with } \\
\text { suppression }\end{array}$ & 4.6 & 8.2 & & 6.2 & 1.5 \\
$\begin{array}{c}\text { Arithmetic with } \\
\text { Trails }\end{array}$ & 8.0 & 10.0 & 6.5 & 1.9 \\
\hline
\end{tabular}

Table 4

Mental Arithmetic Performance as a Function of Number of Carries (With Standard Deviations) in Experiment 2

\begin{tabular}{|c|c|c|c|c|c|c|}
\hline \multirow[b]{3}{*}{ Condition } & \multicolumn{6}{|c|}{ Errors (\%) } \\
\hline & \multicolumn{2}{|c|}{ No Carry } & \multicolumn{2}{|c|}{ One Carry } & \multicolumn{2}{|c|}{ Two Carries } \\
\hline & $M$ & $S D$ & $M$ & $S D$ & $M$ & $S D$ \\
\hline Control & 0 & & 2.1 & 7.1 & 11.8 & 23.6 \\
\hline $\begin{array}{l}\text { Arithmetic with } \\
\text { suppression } \\
\text { Arithmetic with }\end{array}$ & 0 & & 8.3 & 14.1 & 15.6 & 24.2 \\
\hline Trails & 1.0 & 5.1 & 30.2 & 24.4 & 44.8 & 33.0 \\
\hline
\end{tabular}

all the trials. For the Trails task, errors were either omissions or incorrect responses. The next response after an omission was scored as correct if it maintained the original sequence (e.g. A, Monday, -, Tuesday, C, Wednesday). When the original sequence was entirely lost, the next pair of responses was defined as a new reference pair for subsequent responses, in accordance with the task instructions.

A measure of Trails performance on each trial was obtained by expressing the total number of errors as a percentage of the number of intervals. Mean percentage error rates for Trails combined with zero-, one-, and twocarry additions were $10.5,19.6$, and 24.7 , respectively $[F(2,46)=19.74, p<.001]$. Paired contrasts indicated significant increases in Trails errors from zero to one carry and from one to two carries $[F(1,23)<21.10, p<$ .001 , and $F(1,23)=4.97, p<.05$, respectively].

\section{Discussion}

The main aim was to test whether multidigit mental addition uses central executive resources, particularly in relation to carrying. The results give clear support for the first part of this hypothesis, in that the speed and accuracy of calculation were markedly impaired when the full Trails task was performed at the same time. In contrast, suppression had very little effect, confirming the importance of the attention-switching requirement of the Trails task as the locus of interference. Present findings therefore add to the evidence for executive involvement in mental addition (Logie et al., 1994). They also confirm the suggestion that the simplified Trails task used in Experiment 1 did not load the executive effectively.

The hypothesis that executive processes are responsible for carrying is supported by the observation that interference from the Trails task increased with the amount of carrying. Furthermore, Trails performance itself declined systematically as the number of carry operations increased. An alternative interpretation might be that these effects reflect the greater difficulty of adding integers that sum to more than 10 (see, e.g., LeFevre et al., 1996), rather than carrying per se. However, this account would not explain why omitting to carry was by far the predominant type of calculation error.

The nonsignificant effect of articulatory suppression confirmed the results from the continuous presentation condition of Experiment 1. However, on this occasion the 
small increase in calculation errors caused by suppressing articulation approached significance. Moreover, this increase occurred on problems involving carrying, suggesting that the phonological loop could play a minor role in supporting carrying. One speculative possibility is that the loop can be used to store the amount to be carried. For the present set of problems, this amount was always the same, but in general, it is free to vary.

In summary, the full Trails task disrupted the ability to add two continuously visible numbers, but as before, articulatory suppression had very little effect. The detailed pattern of interference between the Trails task and calculation was consistent with the hypothesis that carrying is highly demanding on executive processes.

\section{EXPERIMENT 3}

Although the results of Experiment 2 are compelling, it is possible that the deterioration in Trails performance with number of carries was simply an artifact of the longer solution times associated with carrying. Thus, the Trails task may have been so demanding that performance declined with time on task and not with carry operations per se. A second concern with Experiment 2 is that there was no condition in which the Trails task was performed on its own. This meant that it was not possible to assess whether Trails performance was impaired under dualtask conditions, as would be expected if there is executive involvement in calculation. Experiment 3 therefore addressed these shortcomings by replicating Experiment 2 and adding a condition in which participants performed the Trails task alone. In order to assess whether Trails performance deteriorates as a function of time on task, the Trails-alone condition involved different task durations corresponding to the range of solution times found in Experiment 2.

\section{Method}

\section{Participants}

Twenty-four students from Lancaster University were paid to participate. They were all native English speakers.

\section{Design}

The design was entirely within subjects, the main conditions being mental arithmetic alone, arithmetic with Trails, and Trails alone. In conditions involving arithmetic, there were 12 experimental trials in which the three patterns of carrying (zero, one, or two carries) appeared four times in a random order. There were also 12 experimental trials in the Trails-alone condition. These consisted of 3 trials lasting each of four durations $(15,30,45$, and $60 \mathrm{sec})$, presented in a randomized order. The two Trails conditions were matched for difficulty by using the same set of starting pairs. Dependent variables were the accuracy and latency of mental arithmetic solutions and performance in the Trails task.

\section{Stimuli and Apparatus}

Arithmetic problems were two of the three sets used in Experiment 2 . The apparatus was the same as before, with the addition of a stopwatch for timing trial duration in the Trails-alone condition.

\section{Procedure}

Procedures for the arithmetic-alone and the arithmetic-withTrails conditions were identical to those in Experiment 2. In the Trails-alone condition, the experimenter cued the end of each trial, and there were three practice trials involving a random ordering of the 15-, 30-, and 45-sec durations.

\section{Mental Addition \\ Results \\ Calculation accuracy was again markedly impaired} when the Trails task was performed at the same time $[F(1,23)=18.23, p<0.001]$, and solution times were also much slower $[F(1,23)=12.96, p<.01$; see Table 5]. When accuracy was broken down according to the amount of carrying, the picture was much the same as that in Experiment 2 (see Table 6). Thus, there were very few errors on problems that did not involve carrying, even with the Trails task. Errors increased with the number of carries and did so to a greater extent with the Trails task. As before, Friedman tests were used to assess the effect of carrying on errors. These showed a significant effect of carrying in the arithmetic-with-Trails condition $(p<.001)$ but no significant difference in the control condition.

Calculation errors were classified as before. Taken together, the three defined categories accounted for $67.1 \%$ of all errors. Omitting to carry was by far the most frequent type of error (56.7\%), followed by inappropriate carrying $(9.1 \%)$ and problem/answer substitutions $(1.3 \%)$.

\section{Trails Task}

Trails performance was assessed as in Experiment 2. An initial analysis examined the effect of trial duration when the Trails task was performed alone. Mean percentage error rates for trial durations of $15,30,45$, and $60 \mathrm{sec}$ were $12.1,9.8,11.8$, and 13.5 , respectively. These small differences approached significance $[F(3,69)=2.27, p<$ $.10]$, but there was no evidence for a cumulative effect of time on task.

Table 5

Mental Arithmetic Performance (With Standard Deviations) in Experiment 3

\begin{tabular}{cccccc} 
& \multicolumn{2}{c}{ Errors (\%) } & & \multicolumn{2}{c}{ RT (sec) } \\
\cline { 2 - 3 } \cline { 5 - 6 } Condition & $M$ & $S D$ & & $M$ & $S D$ \\
\hline $\begin{array}{l}\text { Control } \\
\begin{array}{c}\text { Arithmetic with } \\
\text { Trails }\end{array}\end{array}$ & 6.3 & 15.4 & & 5.9 & 1.7 \\
\hline
\end{tabular}

Table 6

Mental Arithmetic Performance as a Function of Number of Carries (With Standard Deviations) in Experiment 3

\begin{tabular}{|c|c|c|c|c|c|c|}
\hline \multirow[b]{3}{*}{ Condition } & \multicolumn{6}{|c|}{ Errors (\%) } \\
\hline & \multicolumn{2}{|c|}{ No Carry } & \multicolumn{2}{|c|}{ One Carry } & \multicolumn{2}{|c|}{ Two Carries } \\
\hline & $M$ & $S D$ & $M$ & $S D$ & $M$ & $S D$ \\
\hline $\begin{array}{l}\text { Control } \\
\text { Arithmetic with }\end{array}$ & 1.0 & 5.1 & 6.3 & 21.2 & 11.5 & 23.3 \\
\hline Trails & 2.1 & 7.1 & 22.9 & 28.5 & 34.0 & 28.9 \\
\hline
\end{tabular}


Next, a comparison was made between overall error rates on the Trails task under single- and dual-task conditions. There was a significant dual-task decrement, with mean error rates of $11.8 \%$ when the Trails task was performed alone and $24.8 \%$ when it was combined with arithmetic $[F(1,23)=36.87, p<.001]$.

In a further analysis, performance on the Trails task was examined as a function of the amount of carrying in the concurrent addition. Mean percentage error rates for problems with zero, one, and two carries were $15.6,25.3$, and 33.7 , respectively $[F(2,46)=27.01, p<.001]$. Pairwise contrasts showed significant increases in error rates between zero and one carry and between one and two carries $[F(1,23)=17.96$ and 12.08 , respectively, $p<.01]$. This substantial effect of carrying replicates Experiment 2 and contrasts with the lack of a task duration effect when the Trails task was performed alone.

A final comparison showed that error rates on the Trails task were slightly but nonsignificantly higher when it was combined with no-carry problems $(15.6 \%)$ than when it was performed alone $[11.8 \% ; t(23)=1.89, p<.10]$.

\section{Discussion}

All the major findings from Experiment 2 were replicated. Thus, arithmetic was markedly impaired by performing the Trails task at the same time, and the impairment was mainly evident on problems involving carrying. The results also confirm the deterioration in performance of the Trails task as the amount of carrying in the arithmetic problems increases.

The most informative new observation is that the effect of time on task in the Trails-alone condition was negligible, as compared with the effect of number of carries when Trails was combined with arithmetic. Therefore, the sensitivity of Trails performance to number of carries is not an artifact of the extra time taken to execute carrying. The second new finding is that the Trails task was performed worse when it was combined with calculation than when it was performed alone, the deterioration being clearly largest when calculations involved carrying. These new findings underpin the conclusion that carry operations involve executive processes. However, the observation of a nonsignificant impairment in Trails performance when there was no carrying in the concurrent calculation suggests (as seems plausible) that executive processes are not confined to carrying.

\section{GENERAL DISCUSSION}

These experiments attempted to map different aspects of the calculation process onto the executive and phonological components of working memory identified in the Baddeley and Hitch (1974) model, as developed by Baddeley $(1986,1996)$. For convenience, the role of the central executive will be addressed first.

Previous research suggested that although working memory is important in mental arithmetic, it does not play a role in carrying (Logie et al., 1994). The present exper- iments manipulated the amount of carrying more systematically and used a different type of interfering task to disrupt executive processes. They began by comparing the interfering effects on calculation of a simplified Trails task and articulatory suppression. However, the simplified Trails task led to no more interference than did suppression, suggesting that it was not a suitable tool for investigating executive processes. Subsequent experiments used the full Trails task as an interfering task, as was originally planned.

The results revealed that the full Trails task caused massive interference with mental arithmetic. Calculation was much slower and less accurate when combined with the Trails task than when performed on its own (Experiments 2 and 3 ) or with articulatory suppression (Experiment 2). Furthermore, performance of the Trails task was disrupted when it was combined with calculation (Experiment 3). The pattern of mutual interference suggested that a major source of difficulty was performing carry operations at the same time as doing the Trails task. This was shown most clearly in the decline in Trails performance as the number of carry operations increased (Experiments 2 and 3 ), an effect that could not be explained in terms of the extra time taken to perform carry operations (Experiment 3). Similarly, there was a consistent tendency for the Trails task to have its greatest disruptive effect on calculations involving carrying (Experiments 2 and 3 ). Finally, it is interesting to note that the majority of calculation errors in Experiments 2 and 3 were carry errors. Overall, therefore, the present results provide strong evidence that the Trails task and carrying compete for common resources. Given previous research on the Trails task (Baddeley, 1996), this competition can be interpreted as evidence that carrying loads executive processes. Previous null results concerning working memory and carrying (Logie et al., 1994) may reflect the use of an insufficiently powerful experimental design.

Carrying was initially regarded as being likely to involve executive control because it involves inhibiting inappropriate but strongly activated operations. Two other possible interpretations are that executive control is required because carrying is unpredictable and/or relatively unpracticed. However, it seems unlikely that the dualtask bottleneck is connected with unpredictability, since each response in the Trails task is entirely predictable from the one before. Lack of practice with the carry operation also seems unlikely, given that the participants were university students. That carrying involves inhibiting strongly primed subroutines, as in the Norman and Shallice (1980) model of executive control, is left as the most promising interpretation. Failure of inhibitory control is consistent with the observation that the most common calculation error was to omit a carry, whereas the converse error of carrying by mistake was much less common. This asymmetry can be readily explained in terms of the greater habit strength of the no-carry schema. However, it is interesting to note that here, too, there are alternative interpretations. For example, one might sup- 
pose that there is some form of mental flag for carrying that is especially vulnerable to being lost. The Trails task would involve two such markers, one for keeping track within each sequence. Conceivably, therefore, there could be a limit on the number of place markers that the executive can track at the same time. Further studies would be needed to distinguish these two accounts. For the present, an interpretation in terms of the Norman and Shallice inhibitory model seems preferable, in view of the other evidence for this approach.

The results also help to clarify the role of the phonological loop in calculation. The hypothesis that the loop is used to store problem information was tested by comparing the interfering effects of articulatory suppression when problems were presented briefly or remained visible. Only in the former case does problem information have to be remembered in order to complete the calculation. The results supported the hypothesis by showing that suppression disrupted arithmetic when problems were presented briefly (Experiment 1; see also Logie et al., 1994) but had very little effect when problems remained visible (Experiments 1 and 2). However, suppression did have a tendency to increase errors on carry problems (see Tables 2 and 4), suggesting that the phonological loop may play a minor role in carrying, perhaps through storing information about the amount to be carried. If so, carrying is not uniquely a function of the central executive.

The question of whether retrieving basic number facts from long-term memory involves executive processes can be addressed by noting that performance on no-carry additions was virtually unimpaired by simultaneously performing the full Trails task (see Tables 4 and 6). This is striking when compared against the substantial interference when additions involved carrying. However, Trails performance was slightly although nonsignificantly impaired when it was combined with no-carry problems (Experiment 3), suggesting that retrieval of arithmetical knowledge may place some demands on executive processes. This is consistent with evidence on the role of working memory in retrieving verbal information from long-term memory (Baddeley et al., 1984) and with the idea that interacting with long-term memory is a function of the executive (Baddeley, 1996). However, another interpretation of the present data is that it is the sequencing of calculation steps that requires executive processes. Further research would be needed to distinguish between these possibilities.

In conclusion, the present findings confirm previous evidence that multidigit arithmetic involves executive and phonological subsystems of working memory (Logie et al., 1994) but go further in identifying different aspects of calculation with the specific subsystems. Thus, it appears that the phonological loop plays a major role when calculation involves storing temporary information, as would be expected on the basis of the Baddeley and Hitch (1974) model of working memory. Carrying operations place a major demand on the central executive, consis- tent with the view that executive control involves inhibiting inappropriate schemata (Norman \& Shallice, 1980). Given the current interest in executive processes, carrying may repay further study as a simple, well-defined example of switching between action schemes in a naturalistic task.

\section{REFERENCES}

Adams, J. W., \& HIrch, G. J. (1997). Working memory and children's mental addition. Journal of Experimental Child Psychology, 67, 21-38.

AshCRAFT, M. H. (1982). The development of mental arithmetic: A chronometric approach. Developmental Review, 2, 213-236.

AsHCRAFT, M. H. (1995). Cognitive psychology and simple arithmetic: A review and summary of new directions. Mathematical Cognition, 1, 3-34.

Ashcraft, M. H., Donley, R. D., Halas, M. A., \& Vakali, M. (1992). Working memory, automaticity, and problem difficulty. In J. I. D. Campbell (Ed.), The nature and origins of mathematical skills (pp. 301-329). Amsterdam: Elsevier.

BADDELEY, A. [D.] (1986). Working memory. Oxford: Oxford University Press.

BADDELEY, A. [D.] (1992). Is working memory working? The fifteenth Bartlett Lecture. Quarterly Journal of Experimental Psychology, 44A, $1-31$.

BADDELEY, A. [D.] (1996). Exploring the central executive. Quarterly Journal of Experimental Psychology, 49A, 5-28.

BadDeley, A. [D.], Lewis, V., EldRidge, M., \& Thomson, N. (1984). Attention and retrieval from long-term memory. Journal of Experimental Psychology: General, 113, 518-540.

BAdDElEY, A. D., \& Hitch, G. J. (1974). Working memory. In G. H. Bower (Ed.), The psychology of learning and motivation (Vol. 8, pp. 47-90). New York: Academic Press.

BADDELEY, A. D., \& LIEBERMAN, K. (1980). Spatial working memory. In R. S. Nickerson (Ed.), Attention and performance VIII (pp. 521539). Hillsdale, NJ: Erlbaum.

Baddeley, A. D., Thomson, N., \& Buchanan, M. (1975). Word length and the structure of short-term memory. Journal of Verbal Learning \& Verbal Behavior, 14, 575-589.

Butterworth, B., Cipolotti, L., \& Warrington, E. K. (1996). Short-term memory impairment and arithmetical ability. Quarterly Journal of Experimental Psychology, 49A, 251-262.

CAMPBELL, J. I. D. (1995). Mechanisms of simple addition and multiplication: A modified network-interference theory and simulation. Mathematical Cognition, 1, 121-164.

ConwaY, A. R. A., \& ENGLE, R. W. (1994). Working memory and retrieval: A resource-dependent inhibition model. Journal of Experimental Psychology: General, 123, 354-373.

Daneman, M., \& Carpenter, P. A. (1980). Individual differences in working memory and reading. Journal of Verbal Learning \& Verbal Behavior, 19, 450-466.

Dansereau, D. F., \& GregG, L. W. (1966). An information processing analysis of mental multiplication. Psychonomic Science, 6, 71 - 72.

DEHAENE, S., \& COHEN, L. (1995). Towards an anatomical and functional model of number processing. Mathematical Cognition, 1, 83-120.

Deloche, G., \& SERON, X. (1987). Numerical transcoding: A general production model. In G. Deloche \& X. Seron (Eds.), Mathematical disabilities: A cognitive neuropsychological perspective (pp. 137170). Hillsdale, NJ: Erlbaum.

FRENSCH, P. A., \& GEARY, D. C. (1993). Effects of practice on component processes in complex mental addition. Journal of Experimental Psychology: Learning, Memory, \& Cognition, 19, 433-456.

Hamilton, J. M. E., \& Sanford, A. J. (1978). The symbolic distance effect for alphabetic order judgements: A subjective report and reaction time analysis. Quarterly Journal of Experimental Psychology, 30, 33-42.

HitcH, G. J. (1978). The role of short-term working memory in mental arithmetic. Cognitive Psychology, 10, 302-323. 
Just, M. A., \& CARPENTER, P. A. (1992). A capacity theory of comprehension: Individual differences in working memory. Psychological Review, 99, 122-149.

LeFevre, J.-A., Bisanz, J., \& Mrkonjic, L. (1988). Cognitive arithmetic: Evidence for obligatory activation of arithmetic facts. Memory \& Cognition, 16, 45-53.

LeFeVre, J.-A., SADESKy, G. S., \& Bisanz, J. (1996). Selection of procedures in mental addition: Reassessing the problem size effect in adults. Journal of Experimental Psychology: Learning, Memory, \& Cognition, 22, 216-230.

LEMAIRE, P., ABDI, H., \& FAYOL, M. (1996). The role of working memory resources in simple cognitive arithmetic. European Journal of Cognitive Psychology, 8, 73-103.

LEZAK, M. D. (1983). Neuropsychological assessment. New York: Oxford University Press.

LogIE, R. H., Gilhooly, K. J., \& WyNN, V. (1994). Counting on working memory in arithmetic problem solving. Memory \& Cognition, 22, 395-410.
MCCloskey, M. (1992). Cognitive mechanisms in numerical processing: Evidence from acquired dyscalculia. Cognition, 44, 107-157.

Norman, D. A., \& Shallice, T. (1980). Attention to action: Willed and automatic control of behavior (Tech. Rep. 99). University of California, San Diego, Center for Human Information Processing.

Shallice, T. (1982). Specific impairments of planning. Philosophical Transactions of the Royal Society of London: Series B, 298, 199-209.

Widaman, K. F., Geary, D. C., Cormier, P., \& LitTle, T. D. (1989). A componential model for mental addition. Journal of Experimental Psychology: Learning, Memory, \& Cognition, 15, 898-919.

Zbrodoff, N. J., \& LoGan, G. D. (1986). On the autonomy of mental processes: A case study of arithmetic. Journal of Experimental Psychology: General, 115, 118-130.

(Manuscript received August 12, 1998; revision accepted for publication August 23, 1999.) 